\title{
Bilateral Ectrodactyly as an Isolated Deformity in a Chromosomally Normal Fetus with an Increased Nuchal Translucency Thickness \\ AM Fernández-Alonso ${ }^{1}$, S García-Solbas ${ }^{1,} \mathrm{~J}$ Berenguel-Rodríguez ${ }^{1}, \mathrm{C}$ Velázquez-Castro del Pino $^{2}$, IM Fernández-Alonso ${ }^{1}$
}

\begin{abstract}
Objective: Finding a bilateral ectrodactyly in association with an increased fetal nuchal translucency, with normal karyotype and genetic studies, binds to do an exhaustive study of all structural fetal anomalies, because although cardiac anomalies are the most frequent, isolated bilateral ectrodactyly can be found.
\end{abstract}

Method: a 35-year-old woman who presents on the first trimester scan a fetal nuchal translucency thickness of $3.7 \mathrm{~mm}$, and throughout gestation the only abnormal fetal finding is a bilateral ectrodactyly.

Results: maternal serum serologies were negative and fetal karyotype was 46XX. The morphologic scan showed a little increased nuchal translucency thickness of $7.8 \mathrm{~mm}$ and bilateral ectrodactyly of the hands with absence of central fingers. Neonate's evolution is normal until 2 years old.

Conclusions: finding an increased fetal nuchal translucency on the first trimester scan raises the need to do an exhaustive study of fetal anatomy, including limbs.

Keywords: Ectrodactyly, nuchal translucency, prenatal diagnosis

From: ${ }^{1}$ Departamento de Obstetricia y Ginecología. Complejo Hospitalario Torrecárdenas. Paraje Torrecárdenas s/n 04009 Almería (Spain)

${ }^{2}$ Unidad de Biotecnología y Genética Clínica. Complejo Hospitalario Torrecárdenas. Paraje Torrecárdenas s/n 04009 Almería (Spain).

Correspondence: Dr AM ${ }^{a}$ Fernández-Alonso, Torrecardenas Hospital, Almería, 04007 Spain. E-mail address: anafernandez.alonso@gmail.com 


\section{INTRODUCTION}

Measurement of fetal nuchal translucency at 11-13+6 weeks of gestation has proven to be an effective screening tool for chromosomal abnormalities (1). Normal fetuses with an increased nuchal translucency have higher incidence of structural anomalies, mainly cardiac, following by renal and abdominal wall, and rare genetic syndromes (2).

Ectrodactyly is estimated to be 1:10.000 to 1:90.000 live births, but it is frequently found in association with other malformations, hence the importance of introducing our case.

\section{CASE REPORT}

A 35-year-old woman, with no medically relevant complaints except an early miscarriage, who present on the first trimester scan a fetal nuchal translucency thickness of $3.7 \mathrm{~mm}$, risk for Down syndrome 1/6 and for Patau syndrome 1/37. Maternal serum serology for toxoplasmosis, rubella virus, cytomegalovirus and herpes virus were negative and fetal karyotype was 46XX. An advance fetal echocardiography was performed with a normal result. The morphology scan showed a little increased nuchal translucency thickness of $7.8 \mathrm{~mm}$ and bilateral ectrodactyly of the hands with absence of central fingers, being the rest of the scan completely normal. Spontaneous labor began at 41 weeks of gestation and cesarean section was performed because of a suspicion of cephalopelvic disproportion. The baby was a 4300 gr woman, Apgar test 9/10. The anomalies that had been described at ultrasound examinations were found (Figure). 


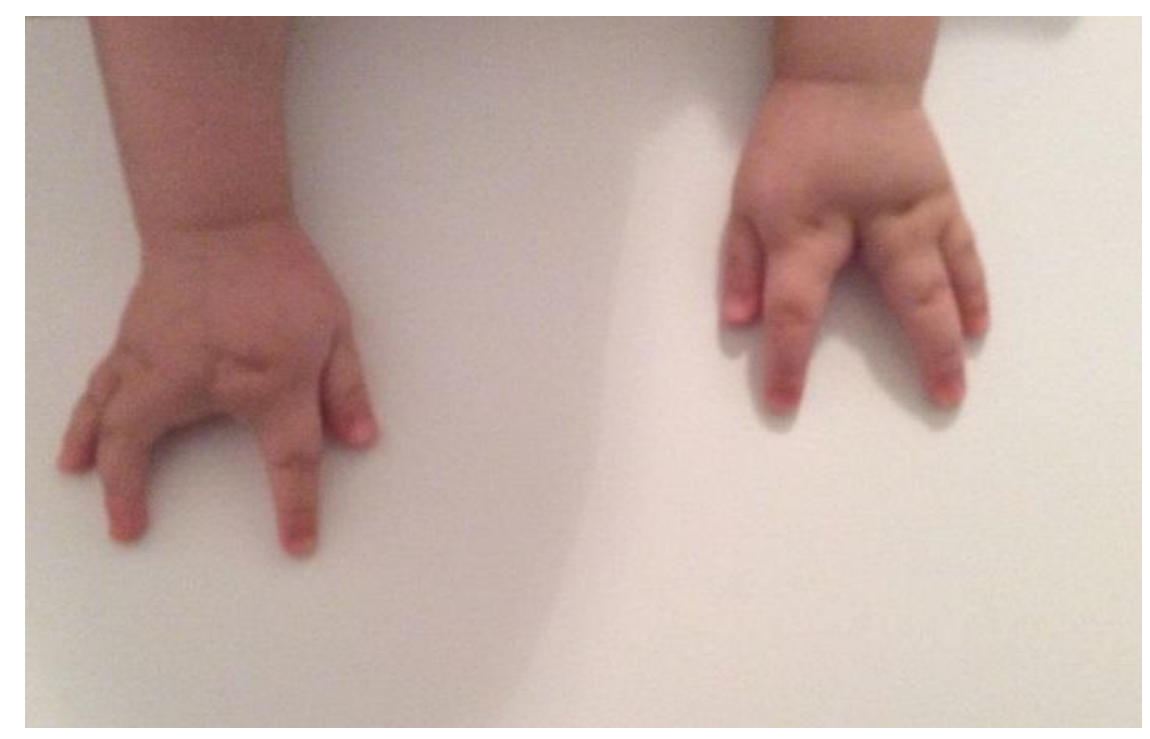

Figure: Bilateral ectrodactyly

Neonate's evolution is normal until 2 years old, with neurologic and motor development also normal. After birth, TP63 gen was studied, finding a mutation c1531 C>A in the exon 12 of TP63 gen in heterozygosis, which has not been described until this moment, being also found at neonate's father, because of what is discarded as a cause of the morphologic anomaly. Mutation of WNT10B gen was also studied, with normal result.

\section{DISCUSSION}

This study confirms the fact that an increased fetal nuchal translucency, further to be a marker for chromosomal abnormalities, is also associated with higher risk of structural anomalies.

Ectrodactyly can be found both in an isolated form and as part of a syndrome. Any of these forms may be sporadic or familiar, being more frequent the sporadic ones (3). The most 
common mode of inheritance is autosomal-dominant with reduced penetrance (4), being more rare X-linked forms.

Ectrodactyly-ectodermal dysplasia-cleft palate syndrome (ECC) is the prototypical example of a syndrome which involves ectrodactyly with multiple organ defects. It is defined as a triad of ectrodactyly, ectodermal dysplasia and cleft lip and palate (5). The EEC syndrome must be distinguished of split-hand/foot malformation (SHFM), which is characterized by syndactyly, median cleft of the hands of feet, and aplasia/hypoplasia of the phalanges, metacarpals and metatarsals. Other syndromes in association with ectrodactyly are Carpenter syndrome, DeLange syndrome, Goltz syndrome and Miller syndrome.

In sporadic ectrodactyly, which is sometimes unilateral, feet are not usually affected. Clinically, there is a deficiency of the three central rays, and the remaining ones may be hypoplastic. It has been postulated than this entity may be the consequence of vascular interruption (6).

\section{CONCLUSION}

Finding a bilateral ectrodactyly in association with an increased fetal nuchal translucency, with karyotype and genetic study both normal, raises the need to do an exhaustive study of the limbs, because this malformation may be present as an isolated form. 


\section{REFERENCES}

1. Pandya PP, Snijders RJM, Johnson SP, Brizot ML, Nicolaides KH. Screening for fetal trisomies by maternal age and fetal nuchal translucency thickness at 10-14 weeks of gestation. Br J Obstet Gynaecol 1995; 102: 957-62.

2. Moscoso G. Fetal nuchal translucency: a need to understand the physiological basis. Ultrasound Obstet Gynecol 1995; 5: 6-8.

3. Duijf P, van Bokhoven H, Brunner HG. Pathogenesis of split-hand/split-foot malformation. Hum Mol Genet 2003; 12: 51-60.

4. Ozen RS, Baysal BE, Devlin B, Farr JE, Gorry M, Ehrlich GD, et al. Fine mapping of the split-hand/split-foot locus (SHFM3) at 10q24: evidence for anticipation and segregation distortion. Am J Hum Genet 1999; 64(6):1646-1654.

5. Tayebi N, Jamsheer A, Flöttmann R, Sowinska-Seidler A, Doelken SC, OehlJaschkowitz B et al. Deletions of exons with regulatory activity at the DYNC1I1 locus are associated with split-hand/split-foot malformation: array CGH screening of 134 unrelated families. Orphanet J Rare Dis. 2014; 9:108.

6. Elliot AM, Evans JA. Genotype-Phenotype Correlations in Mapped Split Hand Foot Malformation (SHFM) Patients. American Journal of Medical Genetics 2006; 140A:1419-1427. 